\title{
Experiential Learning Framework for Design and Development of Environ- mental Data Acquisition System Enhances Student Learning in Undergradu- ate Engineering Courses
}

\author{
Mr. Xavier Shastri Domnique Henry \\ Dr. Lei Zhang, University of Maryland, Eastern Shore
}

Dr. Lei Zhang received his Ph.D. Degree in Electrical Engineering on 2011 from the University of Nevada, Las Vegas. Since 2012 he is working in the Department of Engineering and Aviation Sciences, University of Maryland Eastern Shore. His main research interests include image processing, autonomous system, optical SoC/NoC architecture, and on-chip optoelectronic device design.

\section{Dr. Abhijit Nagchaudhuri, University of Maryland, Eastern Shore}

Dr. Abhijit Nagchaudhuri is currently a Professor in the Department of Engineering and Aviation Sciences at University of Maryland Eastern Shore. He is a member American Society for Mechanical Engineers (ASME), American Society for Engineering Education (ASEE) and, American Society for Agricultural and Biological Engineers(ASABE) and is actively involved in teaching and research in the fields of (i) robotics and mechatronics, (ii)remote sensing and precision agriculture, and,(iii) biofuels and renewable energy. He has published more than 70 refereed articles in journals and conference proceedings. Dr. Nagchaudhuri received his baccalaureate degree from Jadavpur University in Kolkata, India with honors in Mechanical Engineering. Thereafter, he worked in a multinational industry for a little over three years before joining Tulane University as a graduate student in the fall of 1987. He received master's degree from Tulane University in 1989 and doctoral degree from Duke University 1992.

\section{Dr. Madhumi Mitra Ph.D, University of Maryland, Eastern Shore}

Dr. Madhumi Mitra is currently a professor of environmental sciences in the department of Natural Sciences at the University of Maryland Eastern Shore. She is also the coordinator of Biology and Chemistry Education. Dr. Mitra is actively involved in teaching and research in the areas of biofuels and renewable energy; applications of algae in food and environment; and water quality. She has published several peer-reviewed articles in journals and conference proceedings, and is the recipient of various awards and competitive grants. Dr. Mitra received her baccalaureate degree from Presidence College in Kolkata, India with honors in Plant Biology, Geology, and Physiology. She received her master's degree from University of Calcutta, India, and her doctoral degree from North Carolina State University, Raleigh, NC in 2002.

Mr. Christopher E Hartman, University of Maryland, Eastern Shore

Mr. Christopher Alan Toney II, University of Maryland Eastern Shore

Christopher Toney is a Senior Engineering Student at University of Maryland Eastern Shore graduating in Spring 2015. He enjoys the technical aspect of putting a system together to solve engineering problems. When he graduates he hopes to work for a research company as a systems engineer.

Mr. Ayokunle Adeyemi Akangbe A, University of Maryland Eastern Shore

I was born and raised in Nigeria, but came to the United States when I was twelve years old. I am currently a Sophomore at the University of Maryland Eastern Shore majoring in Mechanical Engineering. After obtaining my bachelor's degree, I plan on getting my Master's degree in Mechanical Engineering 


\title{
Experiential Learning Framework for Design and Development of Environmental Data Acquisition System Enhances Student Learning in Undergraduate Engineering Courses
}

\begin{abstract}
Kolb's cycle of experiential learning is a well-known and effective model in education which outlines the process where knowledge is gained through transformative experiences. As students immerse themselves in an active learning framework; acquisition of knowledge results from the combination of participation, assimilation, comprehension and conceptualization of experiential processes in the affective, psychomotor and cognitive domains.

In this paper we outline efforts to integrate Kolb's cycle within the framework of several engineering courses with particular emphases on instrumentation, basic circuits, and programming language courses while involving selected students from these courses to develop a microprocessor based environmental monitoring and data logging system (EMDLS). The data acquisition system developed will be integrated to an Integrated Multi-trophic Aquaculture (IMTA) system and an autonomous boat currently under development at University of Maryland Eastern Shore (UMES).
\end{abstract}

In building the data acquisition system, the engineering students not only get exposure to a crossdisciplinary team of collaborating faculty members from engineering, environmental sciences and aviation programs at the university, but also work closely with graduate students involved in the primary research efforts. The undergraduate students have worked closely with the faculty and graduate students and have followed the system development procedure, where they proposed project objectives, identified design requirements, characterized system specifications, sourced all required components, and are currently involved in system fabrication. The final system is based on the Arduino MEGA and has the capability to measure eight environmental parameters including temperature, color, dissolved oxygen, oxidation reduction potential (ORP), $\mathrm{pH}$, and nitrate levels. While anecdotal evidence can be readily observed from the student excitement and informal feedback, formal assessment tools for documenting learning outcomes are being developed to appraise student learning and will be utilized at the end of the current semester.

\section{Introduction}

A. Motivation of the Project

Increasing demands for cheap protein has led to the degradation of many sensitive ecosystems. As a result, an urgent need exists for the adoption of more sustainable production systems. A viable alternative to terrestrial protein production is aquaculture, of which, shrimp farming is particularly promising. Shrimp farming, usually undertaken in coastal areas, requires fewer inputs and may be done intensively although eutrophication has always been a concern. Culturing algae in tandem 
with such activities, especially nuisance alga like Gracilaria, may offer reprieves including a source of forage, bioremediation services, and possibly, a reliable biofuel feedstock [1-7]. To operate such a system would require significant management and monitoring efforts with respect to wet chemistry and animal husbandry. So automation and instrumentation could offer significant benefit in terms of efficiency and eventual system viability. The efforts outlined in this paper is focused on the development and evaluation of an Arduino controlled and instrumented Integrated Multi-Trophic Aquaculture (IMTA) system based upon the culture of Litopenaeus vannamei and the macro-alga Gracilaria (Figure 1). The platform provides avenues for involving undergraduate engineering students to apply knowledge acquired from courses such as instrumentation and programming languages and support a graduate student in the Food Science and Technology doctoral program with the IMTA development efforts.

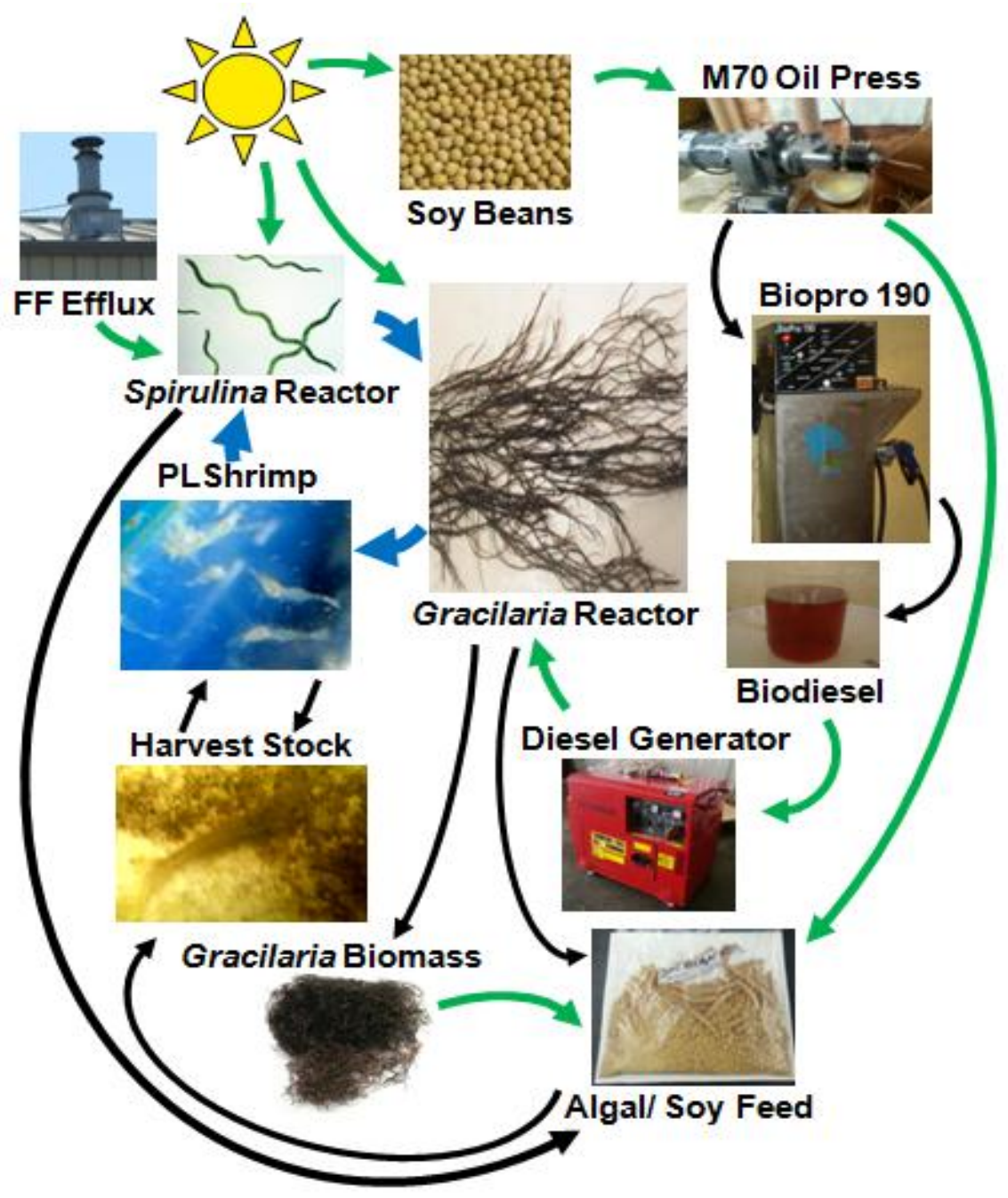

Figure 1. Schematic of the shrimp/ macroalgae IMTA system showcasing the flow of nutrients, water, and energy through the system. 


\section{B. Arduino Boards in Programming Languages and Instrumentation Course at UMES}

Arduino is one of the most popular open-source microprocessor platforms based on easy-to-use hardware and software for building digital devices and interactive objects that can sense and control the physical world $[8,9][1,2]$. It is also widely used as an effective educational tool in many undergraduate engineering courses [10-12]. UMES has an ABET accredited engineering programming with specialization options in mechanical, aerospace, electrical, and computer engineering. All engineering students are required to take Programming Concepts for Engineers (ENGE 170) in freshman/sophomore year and Instrumentation course (ENGE 380) in the junior year where Arduino microprocessor based programming and instrumentation efforts are introduced. Programming in the Arduino Integrated Development Environment (IDE) parallels ' $\mathrm{C}$ ' programming language which is emphasized in ENGE 170. One of the components of the ENGE 380 course is a team project where the students work in groups to develop an innovative instrumentation related project within limited budget. They are allowed to use up to $\$ 20$ to buy additional supplies besides components of "Basic Sensor Kit" available from Manylabs [13] that is provided to them. Student teams are required to demonstrate the understanding of each of the sensors in the "Basic Sensor Kit" using the plug in environment developed by Manylabs, as well as using "Sketch" (C-like programming language for Arduino) before proceeding with their proposed projects. Arduino related efforts are also being integrated in other undergraduate engineering courses related to basic circuits, controls, and microprocessor hardware at UMES. These exposures in coursework provide a foundation for undergraduate students at UMES to effectively participate in the design and development of interdisciplinary projects such as the Arduino-based EMDLS to aid in the management of the day-to-day operations in an experimental Integrated Multi-trophic Aquaculture (IMTA) system under development at UMES.

\section{Experiential Learning Based Educational Research Paradigms}

Experiential Learning Theory (ELT) provides a holistic model which emphasizes the central role that experience plays in the learning process. The most widely used learning theory is Kolb's experiential learning cycle (Figure 2). There are four stages starting with Concrete Experience [14$16]$.

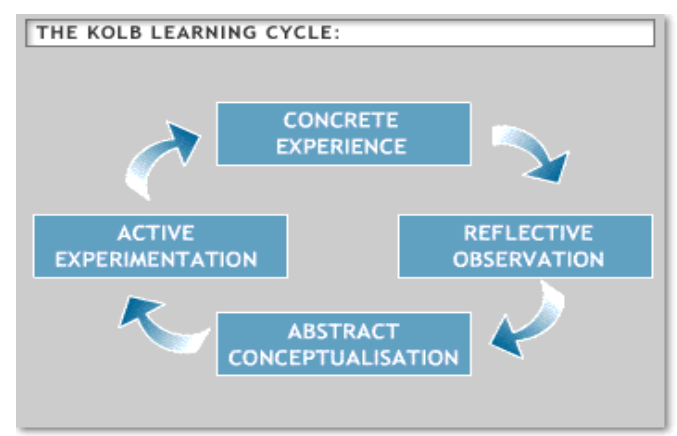

Figure 2. Kolb's cycle of experiential learning[16]. 
According to the Kolb's cycle, the four experiential learning stages are Concrete experience, Reflective observation, Abstract conceptualization and Active experimentation. In this project, the Kolb's Experiential Learning Theory is applied during the EMDLS design and implementation to improve student learning and professional development.

\section{Project Outline}

This project focuses on the design and deployment of a microprocessor based EMDLS to collect environmental data from the 3,000 L raceway tank which forms the basis of the microalgae and shrimp aquaculture system. The data provided by the system will be used in the daily management of the operation as well as to characterize the optimum conditions for the culture of the targeted species. In total, eight water quality parameters will be collected from the tank: $\mathrm{pH}$, water color, rate of flow, temperature, conductivity, dissolved oxygen (DO) level, oxidation reduction potential (ORP) and nitrate level. Each parameter is measured by a separate sensor and data from each sensor will be transmitted to the microprocessor board. To accommodate all eight serial signals from the various sensors, two multiplexers are to be used. At any moment, only one sensor can be selected and allowed to communicate with the microprocessor. The microprocessor will then process the data and store it on an SD card through an SD card shield connected to the microprocessor board. The following system diagram provides a graphical representation of the EMDLS (Figure 3).
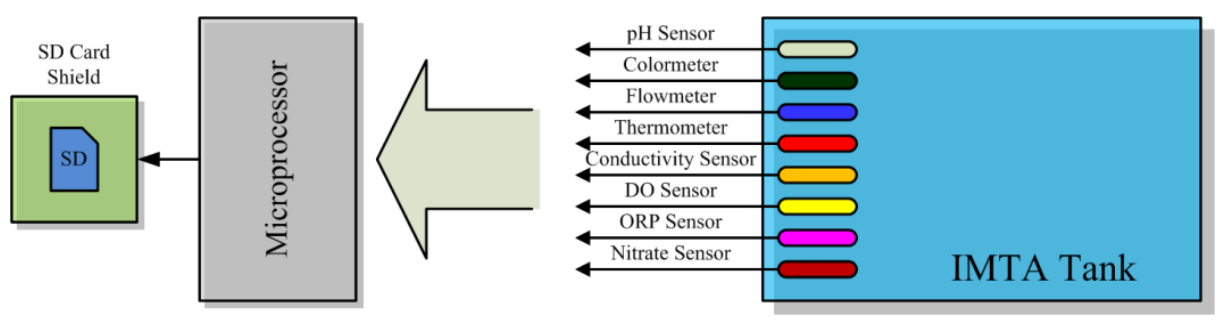

Figure 3. Conceptual diagram of environmental monitoring and data logging

\section{Learning, Development, and Assessment Framework}

Consistent with ABET outcome which requires engineering students to work effectively in multidisciplinary teams, selected undergraduate students are invited to participate in project team meetings related to ongoing cross disciplinary projects led by graduate students and faculty members in engineering, environmental sciences, agriculture, and aviation programs at UMES. Besides IMTA in these team meetings several other efforts related to agricultural automation, automation for environmental monitoring, and UAV based remote sensing are discussed. The exposure provides a rich learning environment for the students. 

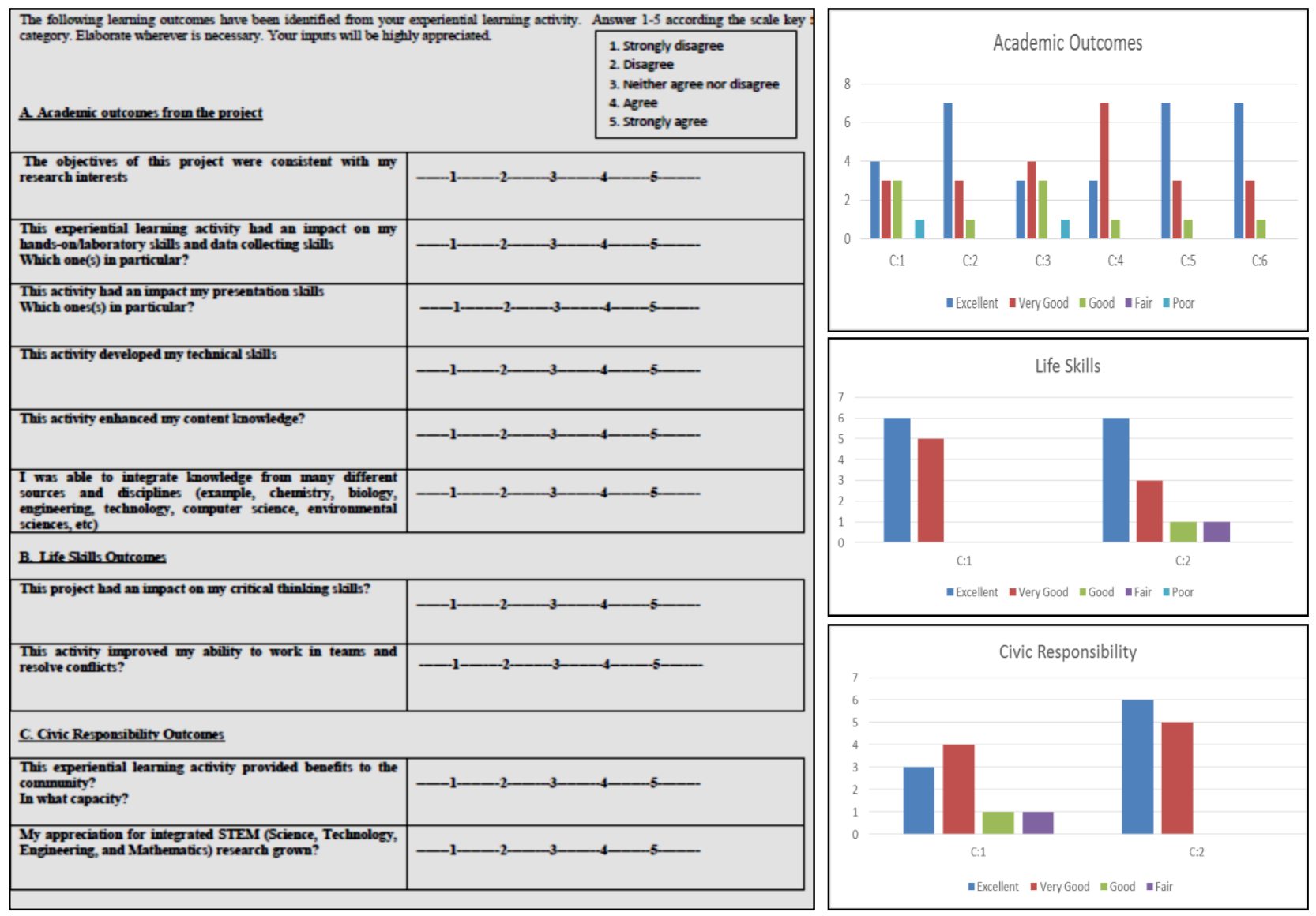

Figure 4. Student Survey Instrument and Assessment Data

Undergraduate students working in the EMDLS project reported here are required to present their progress, share and reflect on their experiences, and get feedback to troubleshoot problems to progress towards the goal through active experimentation, during the weekly meetings.

The students participating in these multidisciplinary team projects are surveyed at the end of every semester to assess the impact the exposure is having on some of the desired academic, life-skills, and civic responsibility outcomes using the survey instrument provided in Figure 4. The bar graphs corresponding to the results of the survey conducted at the end of 2014 fall semester are also shown. The survey results indicate that the students perceive the project experience to be valuable. No attempt has been made here to separate the surveys for the students participating in different projects that are discussed as a team in the cross disciplinary project meetings.

\section{A. Phase I. Acquisition of Sensors and SD Card}

With the support of the lead graduate student, undergraduate engineering students conducted a thorough literature review to gain a better understanding of the environmental parameters that would be critical to the culture of shrimp and macroalgae. The students confirmed the eight aforementioned water quality parameters through literature review as the most critical. Thereafter, 
students conducted an extensive search to find sensors appropriate for the proposed application and to assess the necessary environmental parameters. They identified a nitrate sensor manufactured by PASCO and sourced the remaining sensors from Atlas Scientific. The students also decided to use sensor that would output analog signals for both nitrate and temperature sensing, the rest of the sensors chosen would provide digitalized serial output. A basic SD shield and card in addition to accessory circuits were also purchased for the project. Once all sensors were received, the team tested each sensor with the Arduino MEGA; sensors were connected one by one to the microprocessor and then by utilizing the corresponding manufacturer's codes, each sensor was verified to be in working order.

\section{B. Phase II. Serial Communication}

In Phase II, students worked on the physical connection of all the sensors to the microprocessor to create the first prototype of the EMDLS. Since the selected microprocessor, the Arduino MEGA, only has four hardware Rx/Tx ports (of which only three are usable) for serial communications and six serial signals were needed for the six sensors with serial data output, the team decided to take advantage of the Arduino's Software Serial Interface (SSI). The SSI can create additional serial connections on the regular digital I/O pins [8]. After significant technical literature review and several trial and errors to thoroughly understand SSI implementation, the team successfully incorporated them into the system.

During sensor integration, another problem the team was encountered. Initially, it was decided that a loop would be used in the code to scan all sensors sequentially. However, it was later found that data collected in this way were always incorrectly parsed. Some sensors returned multiple results all at once, while others remained silent for extended periods. After additional research, the team learned that the serial data were treated differently as compared to a regular digital signal on the I/O pin. After the serial port is opened, sensor data arriving at the pin is stored in the serial receive buffer. Therefore access to the correct data from the buffer is only possible with appropriate timing. Based on this observation, the students updated the code by introducing an indicative 'flag' and 'while' loop for each sensor. In this new version of the code, before accessing sensor data a 'flag' will be initialized, under this 'flag' the code will be held by the 'while' loop until the new data from the current sensor is properly received and then the execution will continue. During this stage of the process the students gained experience with the basic serial communication scheme and implemented asynchronous serial communication cases on the Arduino using hard and soft serial ports. The students later learned that not all pins on the Arduino MEGA supported SSI and so there would still be insufficient serial ports for the proposed application. 


\section{Phase III. Multiplexer, A/D and Voltage Divider}

To deal with the shortage of serial communication ports on Arduino MEGA, students finally decided to integrate a multiplexer (MUX) to address this issue. In so doing, they also recognized the additional benefits of using this approach:

- The total number of wires required to connect the microprocessor to all sensors are remarkably reduced. In the original design every sensor required at least two exclusive wires for serial communication, but with the MUX, one pair of Rx/Tx wires is sufficient. This also will result in reduced signal interference and implementation complexity, and increased hardware robustness and reliability.

- Incidentally, students also decided to digitize the two analog sensor signals (nitrate and temperature) to serial data and then transmit the signals to the microprocessor via the MUX. Such analog to digital conversion can reduce the vulnerability of the sensitive analog signals from most additive transmission noises, and thereby greatly improve data reliability.

Digitization of the temperature data was achieved by switching to an existing digital temperature sensor. The digitization of nitrate signal proved more problematic since no similar option was available. After a search of several online resources, the students confirmed that the ORP circuit for the ORP sensor could also be used to digitize the signals from the nitrate sensor. It was also found that the ORP circuit could only digitize voltage signals with a maximum value of $1.5 \mathrm{~V}$, whereas the output from the amplifier of nitrate sensor was in range of 0 to $5 \mathrm{~V}$. To address this problem, the students opted for a simple voltage divider to linearly fit the output from nitrate sensor to the acceptable voltage range for the ORP circuit.

To accommodate all eight serial signals from sensors, two multiplexers (MUXs) (also from AtlasScientific.com) were utlized. At any moment, only one sensor would be selected to communicate with the microprocessor for data acquisition. Every MUX has two digital control pins 'S0' and 'S1'to control the selection of one of the four ports. In addition, every MUX has an active-low 'Enable' control pin 'E'. When the pin 'E' is pulled high, the MUX is disabled. The final system diagram (Figure 5) shows how the digitized signals from the eight sensors are transmitted via the two MUXs to the microprocessor. At this stage of the project, the students were able to demonstrate their knowledge of Digital Logic Design and Basic Circuit Theory.

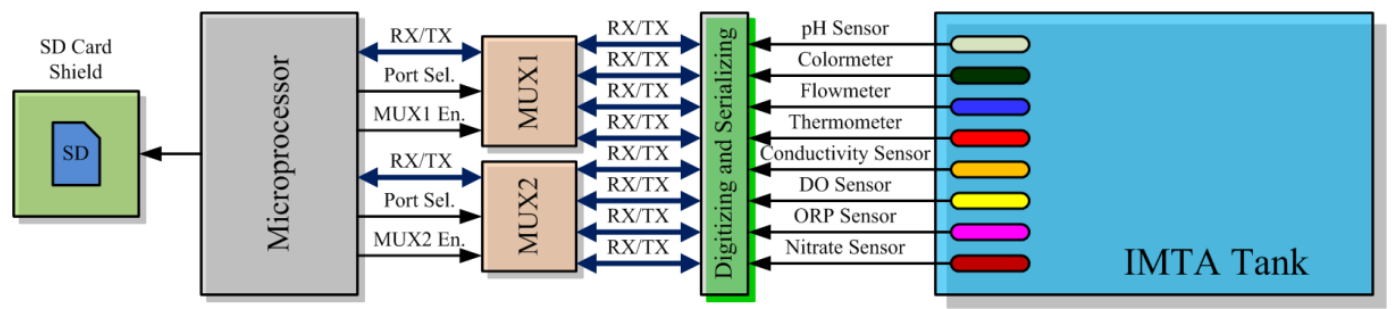

Figure 5. Final EMDLS diagram 


\section{System Implementation and Finalization}

The finalized system layout is the shown in ( See Figure 5, VCC and GND are omitted). The Arduino MEGA microprocessor provides four control signals (MUX1 Enable, MUX2 Enable, and port selection S0 and S1) to choose one of the eight sensors with which to communicate. The output from nitrate sensor via amplifier is sent through a resistive voltage divider to generate the required lower voltage and then sent as one of the eight digitized signals by the ORP circuit.

Between the Arduino MEGA and the sensor, only eight wires were needed, including the Rx/Tx, four control signals, VCC and GND. Students adopted a standard 8-wire Ethernet cable for the connections and as such, an Ethernet cable jack is equipped on the Arduino side for the cable to be plugged in. With this arrangement, the complete sensor kits can be easily removed and reused in other systems, such as the in-development autonomous water surface vehicle for environmental surveillance.

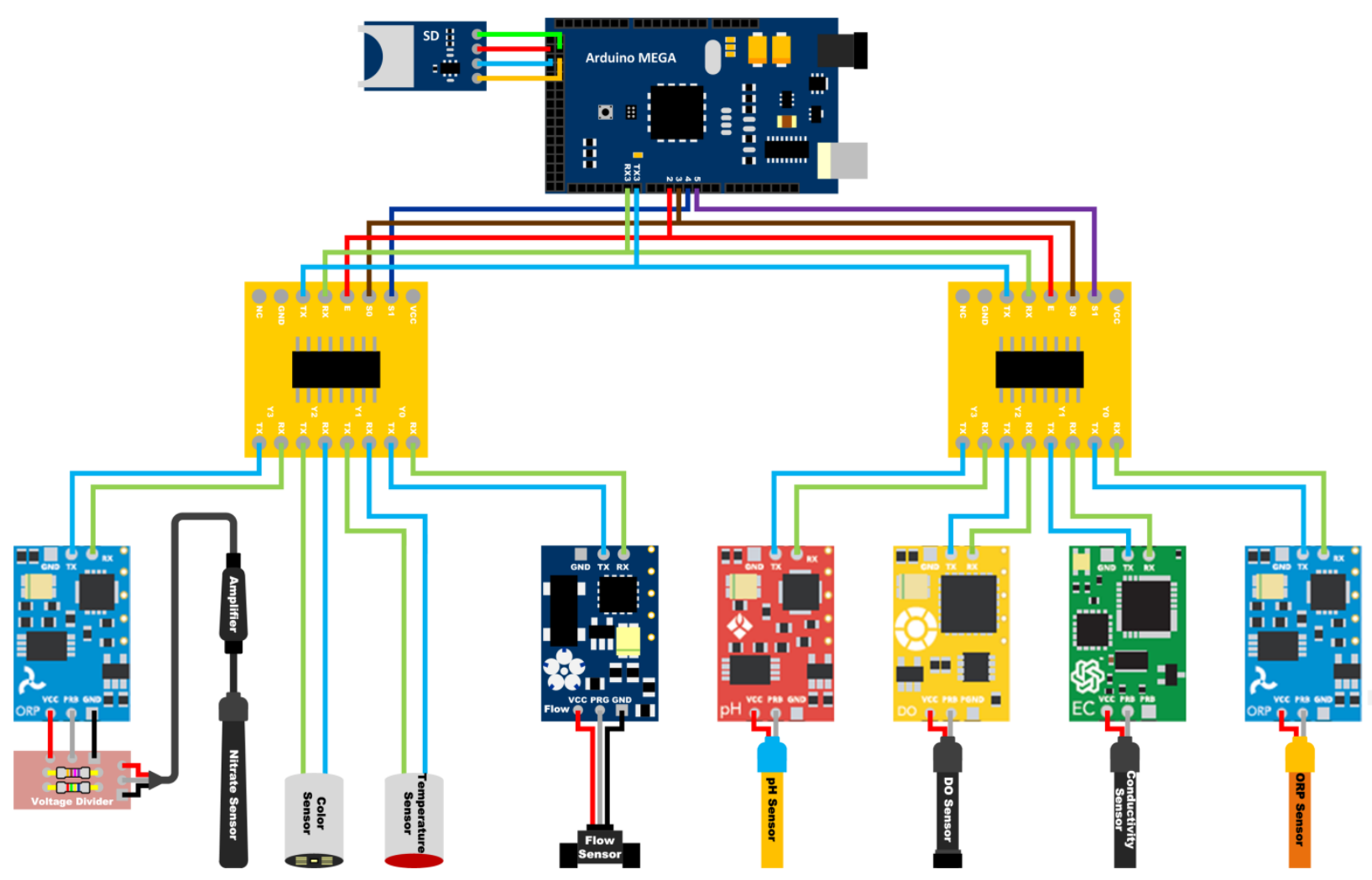

Figure 6. Final EMDLS diagram.

With contributions from the student team and involved faculties, the project objective has been successfully realized. Since testing and implementation (see Figures $6 \& 7$ ), further refinements are underway for the system to be deployed for the continuous monitoring of water quality parameters within the IMTA system. 

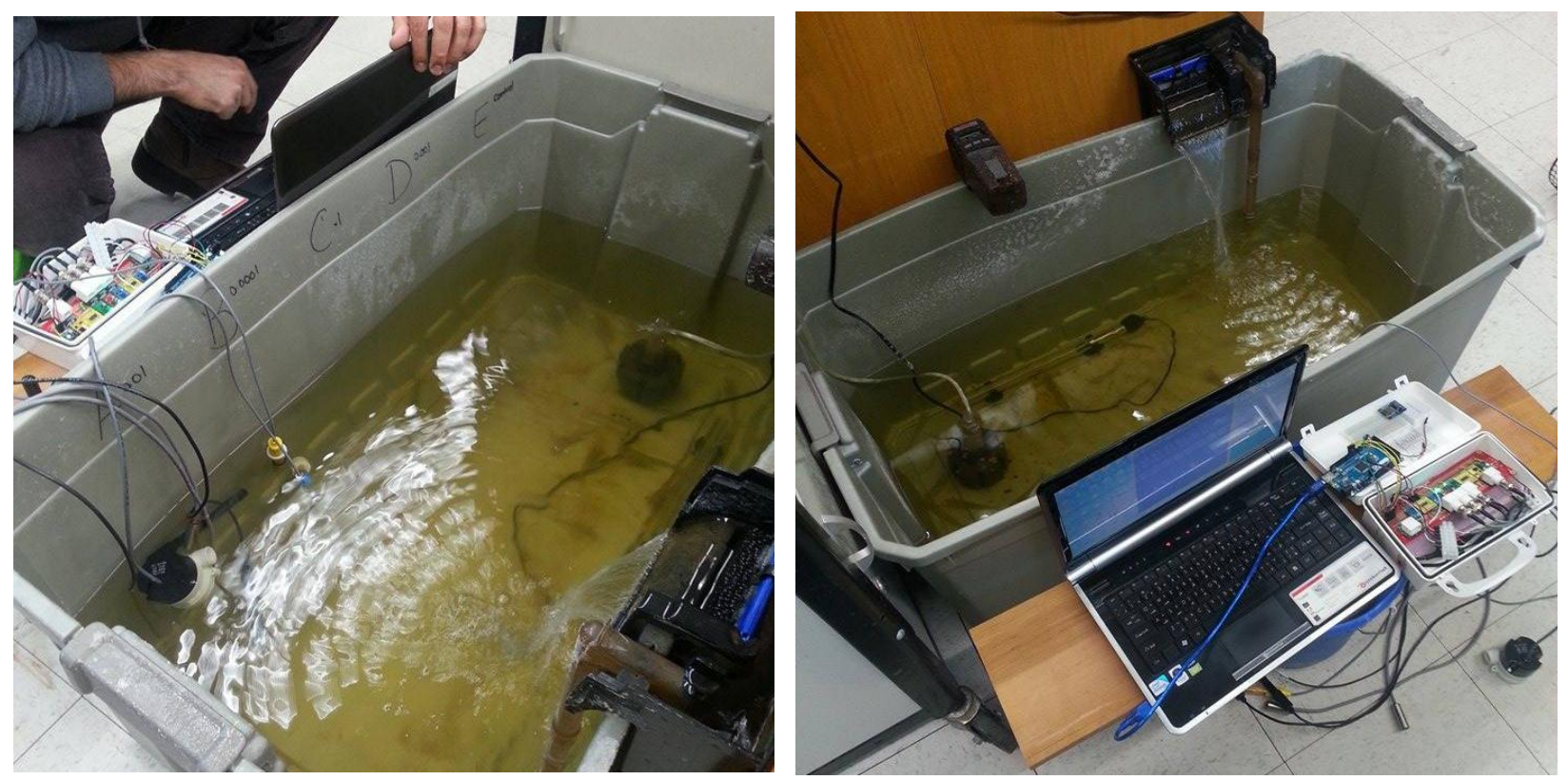

Figure 7. EMDLS testing in temporary shrimp culture tank at the Marine Ecology Lab, UMES.

\section{Future Plans}

Moving forward, the next step for the EMDLS is to improve functionality of the data-logging system to include tasks such as tank environmental control (circulation, heating, etc.), automated feeding system, and camera image-based population estimator kit for the IMTA system. During the preliminary trials the color sensor and the flow sensor did not give proper readings. Investigations are underway in consultation with the vendor to trouble shoot the problems. Also some of the original plans for the completed system has been modified in keeping with observed data integrity issues; students are now planning to read individual sensor data based on user input and display the output on an LCD readout. The sensor-suite will be housed in a student designed enclosure for easy handling and deployment.

\section{Conclusion}

In this paper we presented an Arduino microprocessor based environmental data logger which serves as a component within the IMTA system under development at UMES.

The project was organized, designed and developed by undergraduate engineering students with advice from lead graduate student and faculty. During the process the students went through the experiential learning cycle iteratively, consistent with the Kolb framework by which their comprehension of in-class knowledge was considerably enhanced. Being involved the process of problem identification and directed self-study to find solutions, the students' enthusiasm for learning engineering for real world applications were significantly increased. 


\section{Acknowledgment}

The funds for this project were made available by USDA Evans Allen Grant, USDA Bioenergy and Bioproducts Education Program and Maryland Space Grant Consortium based on proposals submitted by Dr. Mitra and Dr. Nagchaudhuri who serve as PI and Co-I on behalf of UMES and lead the overall effort. Ms. Courtney Shirvani collected the survey data and assisted with the assessment.

\section{Bibliography}

[1] M. Vanthoor-Koopmans, R. H. Wijffels, M. J. Barbosa, and M. H. Eppink, "Biorefinery of microalgae for food and fuel," Bioresource technology, vol. 135, pp. 142-149, 2013.

[2] N. Wei, J. Quarterman, and Y.-S. Jin, "Marine macroalgae: an untapped resource for producing fuels and chemicals," Trends in biotechnology, vol. 31, pp. 70-77, 2013.

[3] K. A. Jung, S.-R. Lim, Y. Kim, and J. M. Park, "Potentials of macroalgae as feedstocks for biorefinery," Bioresource technology, vol. 135, pp. 182-190, 2013.

[4] A. Singh, P. S. Nigam, and J. D. Murphy, "Mechanism and challenges in commercialisation of algal biofuels," Bioresource technology, vol. 102, pp. 26-34, 2011.

[5] M. Aresta, A. Dibenedetto, and G. Barberio, "Utilization of macro-algae for enhanced CO2 fixation and energy production," Prepr. Pap.-Am. Chem. Soc., Div. Fuel Chem, vol. 49, p. 348, 2004.

[6] S. A. Razzak, M. M. Hossain, R. A. Lucky, A. S. Bassi, and H. de Lasa, "Integrated CO2 capture, wastewater treatment and biofuel production by microalgae culturing-A review," Renewable and Sustainable Energy Reviews, vol. 27, pp. 622-653, 11// 2013.

[7] A. d. R. N'Yeurt, D. P. Chynoweth, M. E. Capron, J. R. Stewart, and M. A. Hasan, "Negative carbon via Ocean Afforestation," Process Safety and Environmental Protection, vol. 90, pp. 467-474, 2012.

[8] M. Banzi, Getting Started with arduino: " O'Reilly Media, Inc.", 2009.

[9] J. Oxer and H. Blemings, Practical Arduino: cool projects for open source hardware: Apress, 2009.

[10] N. Bird, "Use of the Arduino Platform for a Junior-Level Undergraduate Microprocessors Course," in American Society for Engineering Education, 2011.

[11] G. Recktenwald and D. Hall, "Using Arduino as a Platform for Programming, Design, and Measurement in a Freshman Engineering Course," in Proceedings of the American Society for Engineering Education Annual Conference \& Exposition, 2011.

[12] G. Tremberger Jr, R. Armendariz, H. Takai, T. Holden, S. Austin, L. P. Johnson, et al., "Applications of Arduino microcontroller in student projects in a community college," in American Society for Engineering Education, 2012.

[13] Basic Sensor Kit with Arduino. Available: https://www.manylabs.org/product/K0001/

[14] D. A. Kolb, Experiential learning: Experience as the source of learning and development vol. 1: Prentice-Hall Englewood Cliffs, NJ, 1984.

[15] R. Loo, "A meta-analytic examination of Kolb's learning style preferences among business majors," Journal of Education for Business, vol. 77, pp. 252-256, 2002.

[16] D. Kolb, "Experiential learning as the science of learning and development," Englewood Cliffs NPH, editor1984, 1984. 